which opens; this arrangement gives little enough light and no air. Can it be healthy? Ought it to be? It is at least most disagreeable.

October 15

\section{ON THE PROPOSED FORTH BRIDGE}

\section{$\mathrm{A}^{\mathrm{N}}$}

$\mathrm{N}$ interesting account of the plan of the railway designed by our distinguished engineer, Mr. Fowler, with the association of Mr. Baker, was given by Mr. Baker to the British Association at their late meeting at Southampton. Supported as it was, to the advantage of those present, by the exhibition of the model of the proposed bridge, it must have given extensive information on the character of the structure. Yet it seems to me that, amidst many valuable particulars, on the strength of materials, their mode of application in this instance, and similar important subjects-it would hardly impress sufficiently, upon the minds of hearers or readers, the vastness of the scheme, the novelty of its arrangements, and the dangers (yet untried) to which, conjecturally, it may be subject. I have thought therefore that I might, without impropriety, offer to the editor of NATURE some remarks on points which after careful consideration have suggested themselves to me. For some particulars I am indebted to the courtesy of Mr. Fowler himself, and I greatly value this kindness.

It is known that at Queensferry the separation of the river-banks, or rather that of the piers next to the banks, at the elevation required for the railway, approaches to a mile. This space is divided by three piers (for which there are excellent foundations on rock and hard clay) into four parts, but only the two middle parts concern us now. They are exactly similar, and are treated in exactly the same way; and subsequent allusions, referring ostensibly to one, are to be considered as applicable to both. Each of the three piers is an iron frame, 350 feet high, the 'central pier 270 feet wide (in the direction of length of the bridge), and each of the others 150 feet. These lofty frames are braced, each upper angle on one side to lower angle on the other side, with no other diagonal bracing, but with a simple tie at mid-height. The lengths of the diagonal bracing are respectively about 430 and 360 feet. The water-spaces between two piers are each about I 700 feet ; and the engineering question now is, how this space of 1700 feet (roughly onethird of a mile) is to be bridged for the passage of a railway.

The plan proposed is, to attach to each side of each frame (that is, to each side which will face a traveller entering upon the bridge) a framed cantilever or bracket about 675 feet long (that is, exceeding in length an English furlong by I 5 feet), attached at top and bottom to the iron frame above mentioned, but having no other support in its.entire length of 675 feet. To give the reader a practical idea of the length of this bracket, I remark that the length of St. Paul's Cathedral, outside to outside, is exactly 500 feet; and thus this bracket, which is to project over the water without any support whatever, is longer than the Cathedral by 175 feet. This in itself is enough to excite some fear, supposing the bracket to support merely its own weight. But further, the bracket bears also the very considerable weight of the roadway and rails. It is also heavily loaded on its point. The two opposing brackets from the two iron frames cover 1350 feet, but the whole space to be covered is I700 feet, leaving 350 feet yet to be supplied for the support of the railway. To furnish this, a lattice-girder carrying a railway is provided, rather more than 350 feet long, whose extremities rest upon the tips of the two brackets.

This statement is enough, I think, to justify great alarm. No specimen, I believe, exists of any cantilever protruding to a length comparable, even in a low degree, to the enormous brackets proposed here. The only structures of this class, in ordinary mechanics, known to $\mathrm{me}$, are the swing-bridges for crossing dock-entrances, and the like, and these are absolutely petty in the present comparison.

I now advert to the weights of the principal portions of the bridge, and the strains which they will create. I understand that the weight of the two parallel braced sides of one bracket is about 3360 tons, to which is to be added the weight of roadway and rails for 675 feet, on which I have no information. I proceed to inquire what strains, in the nature of horizontal pull at the top of the pier and horizontal push at the bottom of the pier, will be caused by this weight. If the weight were evenly dispersed over the triangular bracket, its centre of gravity would be distant from the pier by one-third of the distance of the point from the pier. But as no vertical bar near the pier is included in the weights above, I must take a larger factor, say $\frac{2}{5}$. The vertical weight being 3360 tons, acting at a distance from the pier of $2 \times 675$ feet, and the separation of the points of connection with the pier being 350 feet, it is easily seen that the horizontal pull at the top and push at the bottom are each about 2600 tons. The inclined tension along the great upper bar of the cantilever and the inclined thrust along the great lower bar of the cantilever are therefore each about 2670 tons. The extremities of the great upper bar and the great lower bar being connected at the point of the bracket, and (for a moment) no other weight being supposed to act, there is no tension or thrust at that point, and therefore the tension and the thrust increase gradually, according to the attachment of their loads, from nothing at the point of the bracket to 2670 tons at connection with the pier.

But the point of the bracket is permanently loaded with half the weight of the intermediate 350 -feet railway, or 363 tons, and occasionally loaded with the whole weight of a railway train, say for a passenger train 150 tons (a mineral train would be heavier). The vertical weight of 513 tons thus introduced would be met by a tension of 1004 tons through the whole length of the great upper bar, and a thrust of 1004 tons through the whole length of the great lower bar. Thus we have--

For the great upper bar, a tension increasing from 1004 tons near its point, to 3674 tons near the pier.

For the great lower bar, a thrust increasing from 1004 tons near its point, to 3674 tons near the pier.

The second of these statements particularly requires attention.

Mechanical students and professional engineers are accustomed to estimate by numerical measure the magnitude of a horizontal or nearly horizontal thrust, but persons in ordinary life scarcely attach a clear meaning to such a phrase. I am therefore compelled to make a somewhat violent explanatory supposition, with the hope that it may convey a practical impression as to the meaning of the statements just given.

The great lower bar is in fact a nearly flat frame, braced from side to side, about 120 feet wide at the bottom, and about 40 feet wide at the top, and 690 feet long. Suppose this structure to be planted vertically, say in St. Paul's Churchyard, without any bars, chains, or any thing else, below its vertex, to prevent motion edgewise, but with bracing (which, under ordinary circumstances, would suffice, but which will be the subject of further remark) to prevent its moving flatwise. Its top would be 3 Io feet higher than the top of the cross of St. Paul's Cathedral. Suppose a wtight of 1000 tons to be placed on its very top, and additional weights (if necessary) to be placed at its sides, till the whole weight pressing the ground is 3600 tons. In this state its condition is exactly that of the great lower bar, as regards the crushing and distorting tendency of the weights (although the upper weight itself ought to be considered as partially protected from lateral movement by the great 
upper bar). With this enormous load at this stupendous height, would the citizens of London in the Churchyard below feel themselves in perfect security? I think not; and I claim the same privilege of entertaining the sense of insecurity for the proposed Forth Bridge.

The danger arising from the endwise action of so large a force on so long a bar or frame, is produced by the curvature technically called "buckling," and there appears to be fear of its occurrence in various parts of the bracket, and in some parts sequentially, that is to say. that a buckling of a minor order might lead to a buckling of a more important order. Thus, proceeding from the pier, the first support of the great lower bar is by a suspension-rod from the great upper bar; to which, as regards merely the suspension-rod, there can be no objection. But the upper attachment of this suspensionrod is supported by a thrust-rod about 340 feet long. Can this rod be considered safe against buckling? In the total absence of experiment or explanation, I may be permitted to express a doubt of safety. And if that rod fail, the corresponding part of the great lower bar will sink, it will buckle under its enormous end-thrust, and the bridge will be ruined. The second support of the great lower bar depends, in like manner, on a thrust-rod whose length is 240 feet ; considerations of the same kind apply to it, though probably in a minor degree.

Experienced engineers must have known instances in which buildings have failed from want of consideration of buckling. The following occurred within my knowledge. When the Brunswick Theatre was built, the construction of its trussed iron roof was greatly extolled, and Mr. Whewell and myself, then residing at Cambridge, and proposing to visit London about the same time, had arranged to inspect the truss. But before we reached London it was ruined. There was no adequate bracing of the principal rafters in the plane of the roof; the suspension of a very slight weight on the great tie caused the rafters to buckle sideways, and the roof fell, destroying the building.

I am not aware whether a theory of buckling finds place in any of the books which treat of engineering in a somewhat mathematical form. But there ought to be such. It can be formed with no difficulty and little trouble, giving such a form of result, that all that will be required in any case, to determine the end-pressure which can safely be applied to the end of a bar, will be expressed in terms of the length of the bar, and the curvature caused by a tranversal strain (determined by simple experiment). This theorem ought to be applied in every instance.

I need scarcely to remark that every construction is liable to chance-errors of unforeseen character, and I think that the proposed construction, which depends for its safety entirely on the maintenance of the thrust-principle in perfection, is more liable than any other to danger from these causes. A rivet-head may slip, or a screw may strip, and all may be imperilled. Robert Stephenson, when building the Menai Bridge, used every caution that an active mind could invent: in particular he provided that the masonry for final support of the tubes should be raised as quickly as possible to take the bearing of the tubes at every moment. Yet an accident, though a small one, did happen. The ends of the tubes were raised by the power of hydraulic presses; the cylinder of one of these presses burst, and the end of the tube fell three or four inches. This minute fall, in the judgment of the attendant engineers, gave a strain to the tube such as it never sustained before or since. (This accident came first to my knowledge in a singular way. With the assistance of my friends, Capt. Tupman, R.M.A., and James Carpenter, Esq., and before having heard of the accident, I made experiments on the state of permanent magnetism of the great iron tubes. One of these showed an aromaly, somewhat similar to that of iron heavily struck, On my mentioning this to $\mathrm{Mr}$. Edwin Clark and others, the phenomenon was at once referred to the accidental shock which I have described.)

Much has been said on the action of the wind, and on the difference of that action upon a suspended bridge, and upon a girder bridge. In regard (first) to the amount of pressure, I refer to a former letter of mine, correctly cited in the evidence before the Committee on the fall of the Tay Bridge, in which I state that the maximum pressure may be more than 40 lbs. on the square foot (I should say more than $50 \mathrm{lbs}$. for Scotland), but that this action is so limited, both in time and in local extent [and is, I add, so continually varying in direction], that the average of direct pressure probably would not exceed io lbs. on the square foor. In regard (secondly) to the difference of wind-action in the two systems of construction; - the immediate effect of the wind appears to me to be a shock, of limited extent, which is much less likely to be injurious on a comparatively flexible frame suspended from above, than on a jointed frame where every joint must be tight, and where ruin will follow disturbance. In the proposed Forth Bridge, however, there is risk of danger of the most serious kind, which may perhaps surpass all the other dangers. It arises from the horizontal action of the wind on the great projecting brackets, and its tendency to wrench them laterally from their attachments. The ruinous force depends, not simply on the magnitude of the wind's pressure, but also on its leverage; as measured by the proportion if the height of the Tay Bridge or the length of the bra $\therefore$ et of the Forth Bridge, to the separation (in each case) of their horizontal attachments to the solid piers. This leverage is considerably greater in the instance of the proposed Forth Bridge than it was in that of the unfortunate Tay Bridge; and we may reasonably expect the destruction of the Forth Bridge in a lighter gale than that which destroyed the Tay Bridge.

I may now collect the heads of my remarks on the proposed Forth Bridge :-

I. The proposed construction is, as applied to railway bridges, entirely novel.

II. The magnitude of its parts is enormous.

III. These has been no succession of instances of the construction, with rising degrees of magnitude, which might furnish exferimental knowledge of some of the risks of construction.

IV. The safety of the bridge depends entirely on a system of erd-thrusts upon very long rods; a system which appears generally objectionable, but particularly so when the length of the rods is very great.

$V$. No reference is made to theory applied to the buckling of rods under end-thrusts.

VI. The liability to ruinous disturbance by the lateral power of the wind acting with the leverage of the long brackets appears to be alarmingly great.

My own imfression is, that the proposed construction is not a safe one, and I should be happy to hear that it is withdrawn.

I refer unhesitatingly to "the Suspension Bridge" as the construction which I should recommend. On this system generally I remark: (I) that I am incredulous as to the oscillation of 8 feet in extent, or any sensible part of it ; (2) that if the railway is slightly arched upwards to the degree corresponding to depression caused by an average train, such a train will run on a horizontal plane; (3) that a stiffening lattice may be used with very good effect against vertical oscillations from all causes.

The considerable height of the piers, and the great length of the suspension-chains, are matters to be viewed caref ully.

To reduce them as far as possible, I would sug sest for examination the following proposals :-- 
I. Suppose the stone or iron piers to be much lower than in the plans hitherto proposed, and suppose that the top of a pier carries a bracket on each side, so that the great suspending chain passes over the points of the brackets, and its suspending action begins at those points. The bracket frame may be horizontal where it passes the top of the pier; or it may be raised in a horn on each side, and thus adapted to a smaller height of pier. By this construction, with brackets I 50 feet long (a trifle compared with those of the proposed cantilevers), the piers may without difficulty be shortened 200 feet, and the acting-length of suspending chain may be reduced 150 feet at each end, or 300 feet over each water-channel. This would leave much liberty in regard to the curvature of the chain.

2. It is very desirable, if possible, to reduce the specific weight of the chains per yard, corresponding to a specified suspension strain. This bas been attempted on the Continent by the use of wire, and it has been highly praised for its combination of lightness and strength. The iongest carriage-bridge that I have passed (that of Freyburg, 890 feet span) is a wire bridge. I have also crossed the Rhone at Mo.ntelimart by wire arches of considerable span. I know not whether this construction has been tried in England.

G. B. AIRY

The White House, Greenwich, September 26, 1882

\section{APpENDIX}

Having adverted above to the measurement of the endwise or "buckling" force upon a bar, I will here give a theory, by application of which the admissible amount of end-pressure in any case may be ascertained.

The curvature of any point of a bar depends upon the action of two causes. The first cause is the ex:ernal force, whose angular momentum or effect to bend the bar at any point under consideration is proportional to the produ it of the force (expressed in multiples of a definite unit-as the pound avoirdupois, or the ton, \&c.) by the distance of its line of action from the point under consideration (expressed in multiples of the inch, or the foot, \&c.). The second canse is the internal elastic force of the bar produced by curvature, whose tendency is to oppose the bending action of the external force; I shall assume the magnitude of this force to be proportional to the curvature, or inversely proportional to the radius of curvature, at the point under consideration, its coefficient being for the present expressed only as a symbol. The effects of these two causes balance in a quiescent position of the bar, and they must therefore be made algebraically equal.

The course of investigation will now be as follows : First, I shall give the equation between force and curvature when a bar is bent by a transversal force, acting at the midide of its length. Second, I shall give the similar equation when a bar, at least slightly bent, is exposed to an end-wise force. TThe condition "slightly bent" is necessary to exclude the absurdity of a very heavy weight supported end-ways by a very thin wire.\} In both cases the results will contain the symbolical coefficient to which I have lately alluded. From the first investigation I shall

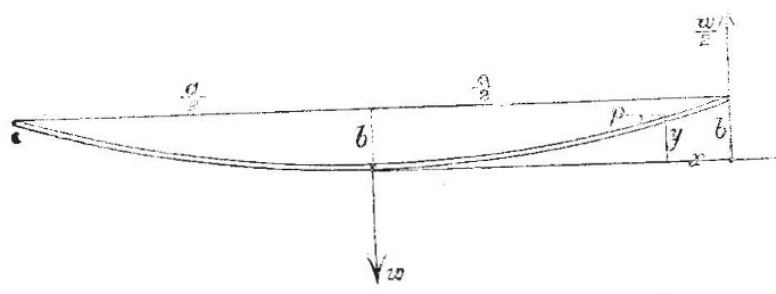

deduce the value of that co-efficient. I shall substitute it in the result of the second investigation; and finally, shall obtain a most convenient expression for the largest admissible force acting endwise on the bar.
(First). Theory of a bar supported at its ends and bent horizontally by a force applied to the middle of its length. The symbols are sufficiently explained in the diagram. It is indifferent, prastically, whether the support of either end of the bar against the force $w$ be a pin (as on the left side), or a force $\frac{w}{2}$ (as on the right side); the latter is the more intelligible. We shall limit our attention to the right-hand half, as the algebraic expressions can be continuous only for the space between two points of application of forces.

Then the angular momentum round the point $p$ produced by the force $\frac{w}{2}$ is $\frac{w}{2} \times x$, tending to throw the point of the bar upwards.

The angular momentum in the opposite direction, produced by the elasticity at $p$, is proportional to $\frac{I}{\text { radius of curvature at } \not p}$, or (if the flexure is not very large) to $\frac{d^{2} y^{\prime}}{d x^{2}}$; and may be called $C \cdot \frac{d^{2} y}{d x^{2}}$, $C$ being the coefficient to which allusion is made above.

Therefore $C \cdot \begin{aligned} & d^{2} y \\ & d x^{2}\end{aligned}$ must $=\frac{w}{2} \times x$, or $\frac{d^{2} y}{d x^{2}}=\frac{w}{2 C} \times x$. Integrating, $\frac{d y}{d x}=\frac{w}{4 C} \times x^{2}+$ constant. To determine the constant, we remark that, when $x=\frac{a}{2}$ the curve is Farallel to the line $a$, or $\frac{d y}{d x}$ is 0 ; and therefore $\frac{w}{4 C} \times \frac{a^{2}}{4}$ + constant $=0$, or constant $=-\frac{w}{4 C} \times \frac{a^{2}}{4} ;$ and the complete value of $\frac{d y}{d x}=\frac{w}{4 C} \times\left(x^{2}-\frac{a^{2}}{4}\right)$. Integrating again, $y=\frac{w}{4 C} \times\left(\frac{x^{3}}{3}-\frac{a^{2} x}{4}\right)+$ new constant. When $x=\frac{a}{2}$, $y$ must $=0$; this gives new constant $=+\frac{a^{3}}{12}$; and the complete value of $y=\frac{w}{4 C} \times\left(\begin{array}{cc}x^{3} \\ 3\end{array}-a^{2} x+\frac{a^{3}}{12}\right)$. This is to equal $b$ when $x=0$, or $\frac{w}{4 C} \times \frac{a^{3}}{12}=b$; from which we obtain $C=\frac{a^{3} \cdot w}{48 \cdot b}$

(Second). Theory of the same bar, at least slightly curved, in a vertical position; its lower end supported on the ground, \&c., and its upper end loaded with a weight $W$.

It will be convenient here to take the centre of length of the vertical line for origin of $x$. As no force or fixation occurs between the two ends of the b.r., the same theory will apply throughout.

Here the angular momentum of the weight $W$ on the point $\not$, tending to bend the top to the right, is $W \times y$. The angular momentum produced by the curvature at $t$, tending to throw the top to the left, is $-C \frac{d^{2} y}{d x^{2}}$. IIt may be convenient to remember that $\frac{d^{2} y}{d x^{2}}$ is here a negative quantity\}. To make these balance we have

$$
W y=-C \cdot \frac{d^{2} y}{d x}, \text { or } \frac{d^{2} y}{d x^{2}}+\frac{W}{C} y=0 .
$$

The solution of this equation is $y=E \cdot \sin \left(x \sqrt{ } \frac{W}{C}\right)+$ $F \cdot \cos \left(x \sqrt{ } \frac{W}{\bar{C}}\right)$ : where $E$ and $F$ must be determined to suit the peculiarities of the case. Now, neglecting the weight of the bar (which may usually be done), the curve will be symmetrical above and below; and therefore the value of $y$ will be the same for $x=+e$ and 
for $x=-e$; $e$ being any number between $-\frac{a}{2}$ and $+\frac{a}{2}$. This cannot hold for $\sin \left(x \sqrt{ }, \frac{W}{C}\right)$, and therefore we must consider $E=0$. The solution therefore is restricted to $F \cdot \cos \left(x \sqrt{ } \frac{W}{C}\right)$. At the centre of the bar, where $x=\mathrm{o}$, this must $=B$. Therefore the solution is $y=B \cdot \cos \left(x \sqrt{\frac{W}{C}}\right)$.

Now here we have a very remarkable circumstance. It will be remembered that in the first investigation we arrived at a relation between $w$, the weight, and $b$, the greatest ordinate of the curve. But bere we find no relation whatever; and we come to this conclusion, that for the state of equilibrium fundamentally assumed, the degree of bulge of the bar is immaterial. And this agrees with plain reasoning : by varying the bulge of the bar, we vary in equal proportions, (I) the elasticity which depends on that bulge and on the general curvature, and (2) the distance of the line of action of $W$ from each point $p$, and its consequent angular momentum; and therefore, if they are equal for one degree of bulge, they

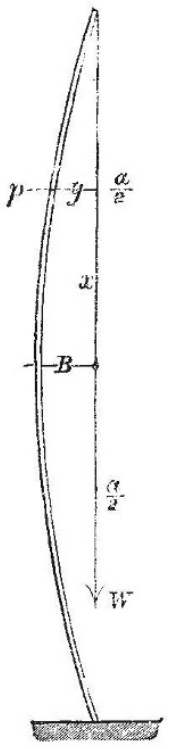

will be equal for every degree of bulge. The value of $B$, therefore is absolutely indeterminate.

But we do obtain one most important conclusion. When $x= \pm \frac{a}{2}, y$ must $=0$. And since, in the product $B \cdot \cos \left(\frac{a}{2} \sqrt{ } \frac{W}{C}\right)$, we are not permitted to make $B$ necessarily $=0$, we must make $\cos \left(\frac{a}{2} \sqrt{ } \frac{W}{C}\right)=0$. The simplest form of effecting this is by making $\frac{a}{2} \sqrt{ }_{C}^{W}=\frac{\pi}{2}$, or $W=C \cdot \frac{\pi^{2}}{a^{2}}$. Substituting for $C$ the value $\frac{a^{3} \cdot w}{48 \cdot b}$, which was found from the first investigation.

$$
W=\frac{\pi^{2}}{48} \cdot \frac{a}{b} \cdot w=0.205 \cdot \frac{a}{b} \cdot w
$$

and this defines the limiting value of the weight under which the curved bar can rest. If the weight be diminished, the curved bar will expand and lift it; if the weight be increased, that increased weight will crush down the curve.

It is important to observe that the furst and second in- vestigations apply to the same bar. And thus, in order to ascertain the limiting buckling force, we need only to ascertain by experiment on the same bar the amount of bend produced by any convenient transversal force.

In some cases, instead of making the first measure by application of the weight $w$ to act horizontally on the middle of the bar, it may be more convenient to make a measure of the vertical flexure of the bar (supported at its two end s in a free horizontal position), produced by its own weight. The following will be the corresponding theory.

(Third). Use the diagram of the first investigation, but substitute $c$ for $b$, and put $Z$ for the whole weight of the bar: and estimate the angular momentum round the point $p$. The reaction upwards of the force $\frac{Z}{2}$ at the pin produces $\frac{Z}{2} \times x$. The action downwards of the weight of bar included between the pin and the point $p$, which is $\frac{Z x}{a}$, will produce $\frac{Z x}{a} \times \frac{x}{2}$ or $\frac{Z x^{2}}{2 a}$. The combination of these produces the angular momentum $Z\left(\frac{x}{2}-\frac{x^{2}}{2 a}\right)$ upwards. The elastic force produces $C \times \frac{d^{2} y}{d x^{2}}$ downwards, where $C$ has the same value as in the first and second investigations. Making these equal, $C \cdot \frac{d^{2} y}{d x^{2}}=\frac{Z}{2 a}\left(a x-x^{2}\right)$. The first integration gives $C \cdot \frac{d y}{d x}=\frac{Z}{a}\left(\frac{a x^{2}}{4}-\frac{x^{3}}{6}\right)+$ ronstant. At the middle of the bar, where $x=\frac{a}{2}, \frac{d y}{d x}$ must $=0$; the constant therefore equals $-\frac{Z}{a}\left(\frac{a^{3}}{16}-\frac{a^{3}}{48}\right)$; and $C \cdot \frac{d y}{d x}=$ $\frac{Z}{a}\left(\frac{a x^{2}}{4}-\frac{x^{3}}{6}-\frac{a^{3}}{24}\right)$.

Integrating again, $C y=\frac{Z}{a}\left(\frac{a x^{3}}{12}-\frac{x^{4}}{24}-\frac{a^{3} x}{24}\right)+$ new constant. This is to be $\circ$ when $x=\frac{a}{2}$; the constant is found to be $+\frac{Z}{a} \cdot a^{4} \cdot \frac{5}{384}$. For the value when $x=0$, and consequently $y=c$, we have $C \cdot c=\frac{5 a^{3} \cdot Z}{384}$, or $C=$ $\frac{5 a^{3} \cdot Z}{384 \cdot c}$ Inserting this value of $C$ in the expression found in the second investigation,

$$
W=\frac{\pi^{2}}{a^{2}} \cdot \frac{5 a^{3} \cdot Z}{384 \cdot c}=\frac{5 \pi^{2}}{384} \cdot \frac{a}{c} \cdot Z=0.128 \frac{a}{c} Z,
$$

where (as before) $W$ is the limit of weight acting endwise on the bar, which the bar can bear without buckling.

If we wish roughly to introduce the consideration of the bar's weight, it will be sufficient to remark that at the lower part of the bar the whole weight of the bar is acting in conjunction with the weight $W ;$ and therefore, when we have computed the force (as above) we ought to deduct from that result the weight of the bar, and the residual will be the force which is permissible for action on the top of the bar.

G. B. A.

\section{THE LATE DR. VAN MONCKHOVEN}

I D Dr. Désiré Charles Van Monckhoven the scientific world has lost an able coadjutor, and his death is to be the more regretted in that he was taken from his many friends when almost in the prime of life. Van Monckhoven was born on September 25, 1834, and on September 25 of this year he died, having thus only traversed forty-eight years of the threescore-and-ten years 